\title{
Cooperative Transmission in Mobile Wireless Sensor Networks with Multiple Carrier Frequency Offsets: A Double-Differential Approach
}

\author{
Kun Zhao, Kegang Pan, and Bangning Zhang \\ College of Communications Engineering, PLA University of Science and Technology, No. 2 Biaoying, \\ Yudao Street, Qinhuai District, Nanjing 210007, China
}

Correspondence should be addressed to Kun Zhao; jucow@sina.com

Received 18 January 2014; Accepted 10 March 2014; Published 3 April 2014

Academic Editor: Linying Xiang

Copyright $\odot 2014$ Kun Zhao et al. This is an open access article distributed under the Creative Commons Attribution License, which permits unrestricted use, distribution, and reproduction in any medium, provided the original work is properly cited.

\begin{abstract}
As a result of the rapidly increasing mobility of sensor nodes, mobile wireless sensor networks (MWSNs) would be subject to multiple carrier frequency offsets (MCFOs), which result in time-varying channels and drastically degrade the network performance. To enhance the performance of such MWSNs, we propose a relay selection (RS) based double-differential (DD) cooperative transmission scheme, termed RSDDCT, in which the best relay sensor node is selected to forward the source sensor node's signals to the destination sensor node with the detect-and-forward (DetF) protocol. Assuming a Rayleigh fading environment, first, exact closed-form expressions for the outage probability and average bit error rate (BER) of the RSDDCT scheme are derived. Then, simple and informative asymptotic outage probability and average BER expressions at the large signal-to-noise ratio (SNR) regime are presented, which reveal that the RSDDCT scheme can achieve full diversity. Furthermore, the optimum power allocation strategy in terms of minimizing the average BER is investigated, and simple analytical solutions are obtained. Simulation results demonstrate that the proposed RSDDCT scheme can achieve excellent performance over fading channels in the presence of unknown random MCFOs. It is also shown that the proposed optimum power allocation strategy offers substantial average BER performance improvement over the equal power allocation strategy.
\end{abstract}

\section{Introduction}

In recent years, with the rapid advances in microelectromechanical systems (MEMS) and wireless communication technologies, wireless sensor networks (WSNs) have gained an increasing research attention for their various military and civil applications, including intrusion detection, automated data collection, healthcare, and environmental monitoring $[1,2]$. A WSN is usually composed of a large amount of low-cost and low-power sensor nodes, which are statically deployed in a certain region of interest. However, in many application scenarios, for example, wildlife protection and object tracking, due to the dynamic changes of events and environment, a purely static WSN could face severe problems, such as limited coverage and channel capacity, unfair energy usage, and increasing multiple missions [3]. To handle these problems, a new class of WSNs, namely, mobile wireless sensor networks (MWSNs), has been proposed by introducing mobility to some or all the sensor nodes, and it is shown that the MWSNs outperform the static WSNs in terms of longer network lifetime, more channel capacity, enhanced coverage and targeting, and so on [3,4]. Many researchers have been dedicated to exploring the aforementioned advantages of MWSNs, and great progress has been made [510]. However, there are still numerous key technical issues that need further research, among which how to realize reliable communications between the mobile sensor nodes over fading channels stands out as critical consideration.

Cooperative communications have been demonstrated as a promising technology to improve the spectral efficiency and reliability of wireless communication systems by forming a virtual antenna array among cooperating nodes [11, 12]. The numerous sensor nodes and the resource-constrained nature make WSNs one of the most important application 
fields for cooperative communications, and a variety of cooperative schemes have been proposed to improve the performance of different kinds of WSNs. These cooperative schemes generally focus on two aspects. On one hand, a number of literatures investigated the cooperative multipleinput and multiple-output (MIMO) transmission technique for WSNs, where the sensor nodes cooperate with each other to form a virtual MIMO channel. The contribution [13] first proposed a cooperative MIMO transmission scheme with Alamouti coding for WSNs, and, based on the similar virtual MIMO, various cooperative schemes employing space-time block codes (STBC) were proposed and analyzed in [1417]. On the other hand, many other researchers endeavored to design excellent selective cooperative relaying schemes. In [18], by combining relay selection with power control, a selective single-relay cooperative scheme was proposed, which can minimize the energy consumption and extend the network's lifetime. A simple geographic-based relay selective cooperative relaying protocol was proposed in [19], where the best relay can be efficiently determined by using the geographical information among nodes. The authors in [20] proposed an adaptive relay selection based cooperative scheme for a cluster-based WSN, which can guarantee the quality of service (QoS) without the needs of prior knowledge of the wireless network model and centralized control.

While all these aforementioned cooperative schemes can significantly improve the performance of WSNs, the key limitation of them is that they all assume full channel state information (CSI) and perfect synchronization can be achieved. However, in actual WSNs, especially MWSNs, where the channels between the sensor nodes undergo different kinds of fading, it is challenging even impossible to obtain perfect CSI. Moreover, as the sensor nodes are evolving towards high mobility, for example, more and more sensors are deployed on ground vehicles and unmanned aerial vehicles $[21,22]$, the perfect synchronization assumption is also not justifiable in MWSNs, where each distributed sensor node is equipped with its local oscillator and the presence of multiple carrier frequency offsets (MCFOs) can be caused by: (i) simultaneous transmissions from spatially separated sensor nodes equipped with different oscillators and (ii) Doppler shifts introduced by the relative motions between the transmit and receive sensor nodes. In such cases, the channels in MWSNs are time-varying; therefore, all these existing cooperative schemes originally developed for static WSNs inevitably suffer drastic performance degradation or even break down.

In order to reduce the burden of channel estimation, noncoherent and differential cooperative transmission schemes have been proposed in $[23,24]$. The works in $[25,26]$ extended the differential modulation to multirelay cooperative networks and showed that full diversity could be achieved. In $[27,28]$ the authors proposed a differential modulation (DM) and relay selection (RS) based scheme for a detect-and-forward (DetF) cooperative network (DM-RSDetF) $[27,28]$, and it was revealed that the DM-RS-DetF network could also achieve full diversity order. However, these schemes still assume that no CFOs exist in the networks, which make them not applicable to the MWSNs with MCFOs.
The approaches for dealing with CFOs in communication systems can be generally classified into two main categories; the first one focuses on estimating and compensating the CFOs by designing excellent estimators, while the second one resorts to developing novel techniques which are robust to the CFOs. Recently, in an effort to eliminate the impact of the MCFOs, a number of MCFOs estimators have been proposed for both amplify-and-forward (AF) and decode-andforward (DecF) cooperative networks [29-32]. Nevertheless, as observed in these literatures, the estimators as well as the transceivers are generally quite computationally complex and the overheads consumed by the parameters estimation are always significant, which limit their deployment on resourceconstrained sensor nodes. Moreover, it is also a challenge task to reliably feedforward/feedback the CSI or MCFOs estimates to different sensor nodes. On the other hand, imperfect MCFOs estimates and compensation still degrade the network performance. Hence, given all these reasons, it may be more practical to address the MCFOs by developing robust transmission techniques rather than by designing estimators to estimate and compensate the MCFOs in actual MWSNs.

In this paper, we consider a MWSN over Rayleigh fading channels, where all the wireless links among the sensor nodes are perturbed by different random MCFOs. This is a practical scenario which has scarcely appeared in the literature. Instead of devising complicated CFO estimator, we propose to address the MCFOs in the MWSN employing doubledifferential (DD) modulation technique, which was originally proposed by Okunev [33]. The major advantage of the DD modulation is its bypass of the CFO and channel estimation, and it has turned out to be a powerful technique to cope with unknown CFOs in a number of cooperative systems [3438]. The AF and DecF based DD cooperative systems were introduced and analyzed in [34] and [35], respectively. The authors in [36] proposed a selective DecF protocol, which could compensate the signal-to-noise ratio (SNR) loss in a single-relay DD cooperative system. To further improve the channel utilization, a low complexity piecewise linear (PL) decoder for the DecF based DD cooperative system was designed in [37], and it was shown that the proposed PL decoder could achieve full spatial diversity, while, in [38], the authors investigated the AF based DD multirelay networks and presented an effective relay selection strategy to improve the system performance.

Motivated by the excellent performance of these schemes, we herein focus on the DetF relaying protocol and develop a robust relay selection (RS) based double-differential cooperative transmission scheme (RSDDCT) to enhance the performance of the MWSN under consideration. In our scheme, a simple and effective Max-Min relay selection strategy is applied to reduce the energy consumption of the network, through which only the best relay sensor is selected to forward the source sensor's double-differential modulated signals to the destination sensor with the DetF protocol. For simplicity, we show MWSN with the proposed RSDDCT scheme RSDDCT-DetF network in the remainder of this paper. To facilitate the performance characterization, we present a comprehensive performance analysis for the 
RSDDCT-DetF network. In this light, we derive exact closedform expressions for the outage probability and average bit error rate (BER), along with their asymptotic expressions in the high SNR regime. Simulation results show that the RSDDCT-DetF network can achieve excellent performance over fading channels in the presence of random MCFOs. While the contributions [34-38] have significantly improved our knowledge on the DD cooperative systems, the most important differences between our work and them are as follows. (1) In [34-37], the authors only focused on single-relay scenarios, whereas WSNs are generally modeled as multirelay networks; moreover, all the analytical results were limited to the error probability and only lower or upper bounds but no exact expressions were derived; (2) In [38], only AF multirelay systems were considered, and the proposed relay selection strategy is not applicable to regenerative networks; in addition, there was no analytical result on the system performance. To the best of the authors' knowledge, there is no previous work on regenerative multirelay cooperative networks with DD modulation. The main contributions of the paper can be summarized as follows.

(i) We propose addressing the MCFOs in MWSNs employing DD modulation technique, which is practical but has not been reported in the literature. More specifically, we develop a robust relay selection based double-differential cooperative transmission scheme, termed RSDDCT, to enhance the performance of MWSNs with MCFOs.

(ii) Assuming a Rayleigh fading environment, we derive exact closed-form expressions for the outage probability and average BER of the RSDDCT-DetF network at arbitrary SNRs, which provide a fast and efficient means to evaluate the performance of the MWSNs employing the proposed scheme.

(iii) In order to gain further insights into the impact of system parameters, such as fading parameters and number of relay sensors, we look into the high SNR regime and present simple and informative high SNR approximations for the outage probability and average BER, which reveal that the MWSN with the proposed scheme can achieve full diversity order.

(iv) Based on the derived analytical expressions, we formulate an interesting optimization problem which seeks to minimize the average BER. In particular, we consider power allocation among the source sensor and the relay sensors under a total transmit power constraint, and simple closed-form solutions are derived. Comparisons based on simulations demonstrate that a significant performance improvement is achieved using the optimum power allocation compared to the equal power allocation, which provide an effective method to improve the MWSN's performance under a fixed power budget.

The rest of the paper is organized as follows. Section 2 introduces the DD modulation and the system model of the RSDDCT-DetF network. In Section 3, we derive closed-form expressions for the outage probability and average BER of the

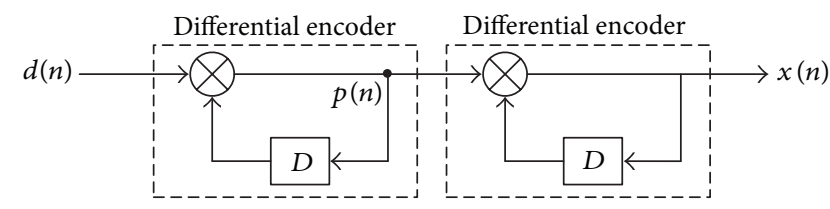

(a)

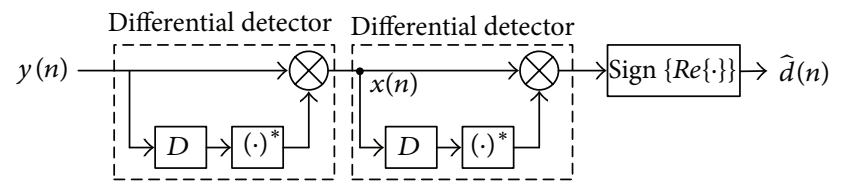

(b)

Figure 1: Double-differential (a) modulator and (b) demodulator.

RSDDCT-DetF network. The asymptotic system behaviors and the power allocation strategy are provided in Sections 4 and 5, respectively. Finally, Section 6 presents our numerical results, and Section 7 concludes the paper.

\section{System Model}

2.1. Double-Differential Modulation. We suppose that double-differential binary phase shift keying (DDBPSK) modulation is utilized in the network. Let $d(n) \in\{+1,-1\}$ denote the unitary symbols belonging to the BPSK constellation to be transmitted in the $n$th symbol interval. As shown in Figure 1(a), in a DD modulation based system, the double-differential modulated signals $x(n)$ are obtained from $d(n)$ in the following way:

$$
\begin{aligned}
& p(n)=p(n-1) d(n), \\
& x(n)=x(n-1) p(n), \quad n=1,2, \ldots, L,
\end{aligned}
$$

where $p(0)=x(0)=1$ and $L$ is the number of the symbols to be sent within a frame.

Consider a fading channel with random CFO; the received signals can be expressed as

$$
y(n)=\sqrt{P} e^{j \omega n} h x(n)+z(n),
$$

where $P$ is the transmit power, $h$ is the channel fading coefficient, $\omega \in[-\pi, \pi)$ is the unknown normalized random $\mathrm{CFO}$ in radians, and $z(n) \sim \mathrm{CN}\left(0, N_{0}\right)$ is the additive white Gaussian noise (AWGN), with $C N\left(\mu, \sigma^{2}\right)$ representing a complex Gaussian random variable with mean $\mu$ and variance $\sigma^{2}$.

The optimal decoder for DDBPSK signals is the maximum likelihood decoder (MLD), which is given by [39]

$$
\begin{aligned}
\widehat{d}(n) & =\operatorname{sign}\left\{\operatorname{Re}\left\{\left(y(n) y^{*}(n-1)\right)\left(y(n-1) y^{*}(n-2)\right)^{*}\right\}\right\} \\
& =\operatorname{sign}\left\{\operatorname{Re}\left\{X(n) X^{*}(n-1)\right\}\right\},
\end{aligned}
$$

where $X(n)=y(n) y^{*}(n-1)$. It is noted from (3) that the MLD for DD signals can be regarded as a differential detector for the equivalent single-differential signals $X(n)$, which is clearly 
depicted in Figure 1(b). Thus, the BER performance of DD signals can be characterized by $X(n)$, which is given by

$$
\begin{aligned}
X(n)= & P|h|^{2} e^{j(\omega+\Delta \phi(n))}+\sqrt{P} e^{j \omega n} h x(n) z^{*}(n-1) \\
& +\sqrt{P} e^{-j \omega(n-1)} h^{*} x^{*}(n-1) z(n)+z(n) z^{*}(n-1),
\end{aligned}
$$

where $\Delta \phi(n)=\arg (x(n))-\arg (x(n-1))$. It is shown that the instantaneous SNR of $X(n)$ can be approximated as

$$
\gamma_{X} \approx \frac{\gamma_{y}}{2+\gamma_{y}^{-1}} \approx \frac{\gamma_{y}}{2}-\frac{1}{4},
$$

where $\gamma_{y}=|h|^{2} P / N_{0}$. Based on the SNR approximation, the BER performance of the DDBPSK modulation can be evaluated as [34]

$$
P\left(\gamma_{y}\right)=\frac{1}{2} \exp \left(\frac{\gamma_{y}}{2}-\frac{1}{4}\right)
$$

2.2. RSDDCT-DetF Mobile Wireless Sensor Networks. Consider a MWSN as shown in Figure 2(a), where a source sensor node $(S)$ communicates with a destination sensor node $(D)$ with the assistance of a number of potential relay sensor nodes $R_{i}\left(i=1, \ldots, N_{R}\right)$. We suppose that the channel coefficients $h_{S D}$ (between $S$ and $D$ ), $h_{S R}^{i}$ (between $S$ and $R_{i}$ ), and $h_{R D}^{i}$ (between $R_{i}$ and $D$ ) are all flat Rayleigh fading coefficients. In addition, $h_{S D}, h_{S R}^{i}$, and $h_{R D}^{i}$ are mutually independent and nonidentical. It is assumed that all the links are perturbed by independent random CFOs $\left(\omega_{S D}, \omega_{S R}^{i}\right.$, and $\left.\omega_{R D}^{i}\right)$ and the fading coefficients keep constant within one frame and independently change from one frame to another, which are modeled as $h_{S D} \sim C N\left(0, \sigma_{1}^{2}\right), h_{S R}^{i} \sim C N\left(0, \sigma_{2}^{2}\right)$, and $h_{R D}^{i} \sim C N\left(0, \sigma_{3}^{2}\right)$. We also assume here that all the AWGN terms of all links have zero mean and equal two-sided spectral density $\left(N_{0} / 2\right)$. The transmit powers of the source sensor and each relay sensor are $P_{1}=\lambda P$ and $P_{2}=(1-\lambda) P$, respectively, where $P=P_{1}+P_{2}$ denotes the total transmit power of the network and $\lambda \in(0,1)$ is the power allocation factor. In this respect, the MWSN under consideration can be further described by a more informative block model, which is presented in Figure 2(b).

Suppose that each transmission frame is of length $2 L$, where $L$ is the number of the data symbols transmitted from each sensor node within a frame. During the first phase, $S$ broadcasts a stream of signal sequence of length $L$ to all the relay sensors and $D$; the received symbols at the $i$ th relay sensor and $D$ can be expressed as

$$
\begin{aligned}
& y_{S R}^{i}(n)=\sqrt{P_{1}} h_{S R}^{i} e^{j \omega_{S R}^{i} n} x(n)+z_{S R}^{i}(n), \\
& y_{S D}(n)=\sqrt{P_{1}} h_{S D} e^{j \omega_{S D} n} x(n)+z_{S D}(n),
\end{aligned}
$$

where $\omega_{S R}^{i}$ and $\omega_{S D}$ are the random CFOs; $z_{S R}^{i}(n)$ and $z_{S D}(n)$ denote the AWGN at the $i$ th relay and $D$, respectively. In this paper, we assume that all the CFOs follow uniform distribution and remain constant over at least three consecutive symbol intervals. However, it should be pointed out

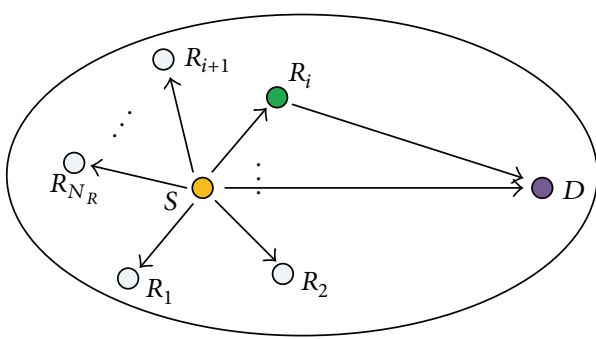

(a)

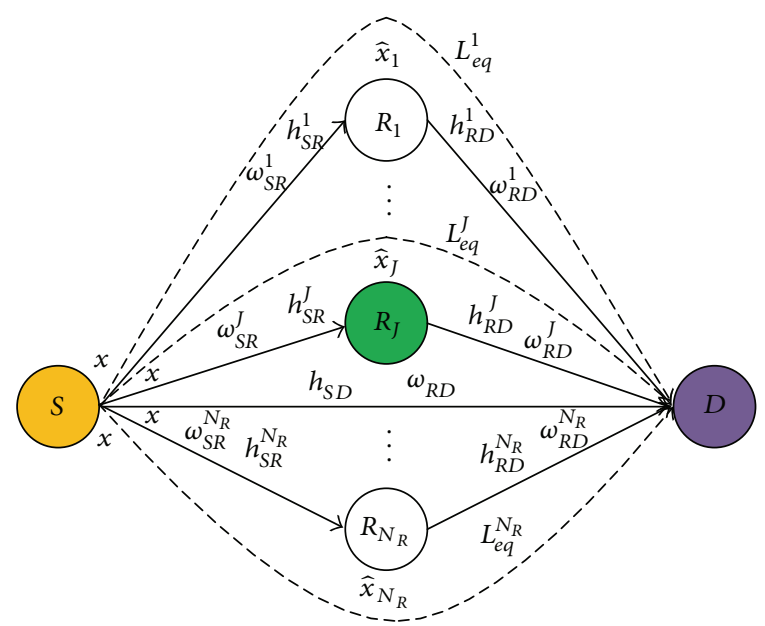

(b)

FIGURE 2: A cooperative MWSN with relay selection. (a) System structure. (b) Block model.

that, in general, there is no restriction over the probability distribution of the CFOs and they could have any probability distribution.

In the second phase, only the relay sensor with the best link is selected to forward the remodulated signals to $D$. Assuming that relay sensor $R_{J}$ is selected, the received symbols at $D$ can be written as

$$
y_{R D}^{J}(n)=\sqrt{P_{2}} h_{R D}^{J} e^{j \omega_{R D}^{J} n} \widehat{x}_{J}(n)+z_{R D}^{J}(n),
$$

where $\omega_{R D}^{J}$ denotes the CFO of the second-link, $\widehat{x}_{J}(n)$ represents the remodulated symbols at $R_{J}$, and $z_{R D}^{J}(n)$ is the AWGN of the selected $R_{J}-D$ link.

To take into account the detection errors at the relay sensors, we apply the one-hop equivalent link model developed in [25]. As shown in Figure 2(b), the equivalent one-hop links are denoted by $L_{e q}^{i}, i=1, \ldots, N_{R}$, and the SNR of each equivalent link can be approximated as

$$
\gamma_{e q}^{i} \approx \min \left\{\gamma_{S R}^{i}, \gamma_{R D}^{i}\right\}
$$

where $\gamma_{S R}^{i}=\lambda \bar{\gamma}\left|h_{S R}^{i}\right|^{2}$ and $\gamma_{R D}^{i}=(1-\lambda) \bar{\gamma}\left|h_{R D}^{i}\right|^{2}$ are the instantaneous SNR of the $S-R_{i}$ and $R_{i}-D$ links, respectively, with $\bar{\gamma}=P / N_{0}$ denoting the average SNR. It is worth pointing out that the same bounding technique has been widely adopted in the performance analysis of various relay systems; see $[25,27,28]$ and references therein. In addition, 
it has been demonstrated that this lower bound is in general very tight for a wide range of SNR.

Based on the equivalent links, the best relay sensor $R_{J}$ is selected according to the Max-Min criterion given by

$$
J=\arg \max _{1 \leq i \leq N_{R}} \min \left\{\gamma_{S R}^{i}, \gamma_{R D}^{i}\right\},
$$

where the instantaneous SNR of the worst one of the twohop relay link is maximized. To perform relay selection at the relay sensors, the source sensor and destination sensor should send request-to-send (RTS) and clear-to-send (CTS) packets before each transmission, respectively. Based on the RTS and CTS packets, each relay sensor first estimates the amplitudes of the channels from the source and destination sensors to calculate $\gamma_{S R}^{i}, \gamma_{R D}^{i}$ and the equivalent SNR $\gamma_{e q}^{i}$; then a backoff timer is set to be inversely proportional to $\gamma_{e q}^{i}$; therefore, the best relay sensor with the largest equivalent SNR has the smallest backoff time and consequently occupies the channel to forward its signals to the destination sensor, and all other relay sensors keep silent in this transmission interval.

In order to efficiently combine the two received signals, we propose a new linear combiner to process the DD signals, where the two branches are combined as

$$
\begin{aligned}
y_{C}(n)= & \left(y_{S D}(n) y_{S D}^{*}(n-1)\right)\left(y_{S D}(n-1) y_{S D}^{*}(n-2)\right)^{*} \\
& +\left(\frac{\gamma_{e q}^{J}}{\gamma_{R D}^{J}}\right)^{2}\left(y_{R D}^{J}(n) y_{R D}^{J}{ }^{*}(n-1)\right) \\
& \times\left(y_{R D}^{J}(n-1) y_{R D}^{J}{ }^{*}(n-2)\right)^{*},
\end{aligned}
$$

in which the weights of the two branches are chosen to maximize the output SNR of $y_{C}(n)$ [25] and $\gamma_{e q}^{J}$ and $\gamma_{R D}^{J}$ denote the equivalent SNR and second-hop SNR of the selected link, respectively. After combination, the total output SNR can be rewritten as

$$
\gamma_{T}=\gamma_{S D}+\gamma_{e q}^{J}
$$

where $\gamma_{S D}$ is the instantaneous SNR of the direct link.

According to (3), the combined signals can be rewritten as

$$
\begin{aligned}
y_{C}(n)= & \left(X_{S D}(n) X_{S D}^{*}(n-1)\right) \\
& +\left(\frac{\gamma_{e q}^{J}}{\gamma_{R D}^{J}}\right)^{2}\left(X_{R D}^{J}(n) X_{R D}^{J}{ }^{*}(n-1)\right),
\end{aligned}
$$

where $X_{S D}(n)=y_{S D}(n) y_{S D}^{*}(n-1)$ and $X_{R D}^{J}(n)=$ $y_{R D}^{J}(n) y_{R D}^{J}{ }^{*}(n-1)$, and the detection of the transmitted symbols is performed as

$$
\widehat{d}(n)=\operatorname{sign}\left\{\operatorname{Re}\left\{y_{C}(n)\right\}\right\} .
$$

\section{Performance Analysis}

In this section, we present a comprehensive performance analysis for the RSDDCT-DetF network described in the previous section. Specifically, we derive exact closed-form expressions for two important performance metrics, that is, outage probability and average BER.

3.1. Preliminaries. Before delving into the detailed analysis, we first present the statistical behaviors of $\gamma_{S D}$ and $\gamma_{e q}^{J}$, which will be frequently invoked in the subsequent derivations.

As all the channels undergo Rayleigh fading, the PDFs and CDFs of $\gamma_{S D}, \gamma_{S R}^{i}$, and $\gamma_{R D}^{i}$ can be written as [40]

$$
\begin{array}{cc}
p_{\gamma_{S D}}\left(\gamma_{S D}\right)=\frac{1}{\bar{\gamma}_{S D}} e^{-\gamma_{S D} / \bar{\gamma}_{S D},} & F_{\gamma_{S D}}\left(\gamma_{S D}\right)=1-e^{-\gamma_{S D} / \bar{\gamma}_{S D},} \\
p_{\gamma_{S R}^{i}}\left(\gamma_{S R}^{i}\right)=\frac{1}{\bar{\gamma}_{S R}} e^{-\gamma_{S R}^{i} / \bar{\gamma}_{S R}}, & F_{\gamma_{S R}^{i}}\left(\gamma_{S R}^{i}\right)=1-e^{-\gamma_{S R}^{i} / \bar{\gamma}_{S R}}, \\
p_{\gamma_{R D}^{i}}\left(\gamma_{R D}^{i}\right)=\frac{1}{\bar{\gamma}_{R D}} e^{-\gamma_{R D}^{i} / \bar{\gamma}_{R D}}, & F_{\gamma_{R D}^{i}}\left(\gamma_{R D}^{i}\right)=1-e^{-\gamma_{R D}^{i} / \bar{\gamma}_{R D}},
\end{array}
$$

where $\bar{\gamma}_{S D}=\lambda \sigma_{1}^{2} \bar{\gamma}, \bar{\gamma}_{S R}=\lambda \sigma_{2}^{2} \bar{\gamma}$, and $\bar{\gamma}_{R D}=(1-\lambda) \sigma_{3}^{2} \bar{\gamma}$. Using (16) as a starting point, we can derive the statistic properties of $\gamma_{e q}^{J}$ as the following lemma.

Lemma 1. The PDF and $C D F$ of $\gamma_{e q}^{J}$ are given by

$$
\begin{aligned}
p_{\gamma_{e q}^{J}}\left(\gamma_{e q}^{J}\right) & =\frac{N_{R}}{\bar{\gamma}_{e q}}\left(1-e^{-\gamma_{e q}^{J} / \bar{\gamma}_{e q}}\right)^{N_{R}-1} e^{-\gamma_{e q}^{J} / \bar{\gamma}_{e q}} \\
& =\frac{N_{R}}{\bar{\gamma}_{e q}} \sum_{k=0}^{N_{R}-1} C_{N_{R}-1}^{k}(-1)^{k} e^{-(k+1) \gamma_{e q}^{J} / \bar{\gamma}_{e q}}, \\
F_{\gamma_{e q}^{J}}\left(\gamma_{e q}^{J}\right) & =\left(1-e^{-\gamma_{e q}^{J} / \bar{\gamma}_{e q}}\right)^{N_{R}},
\end{aligned}
$$

where $\bar{\gamma}_{\text {eq }}=\left(\lambda(1-\lambda) \sigma_{2}^{2} \sigma_{3}^{2} /\left(\lambda \sigma_{2}^{2}+(1-\lambda) \sigma_{3}^{2}\right)\right) \bar{\gamma}$ and $C_{N_{R}-1}^{k}$ denotes the binomial coefficient.

Proof. As $\gamma_{e q}^{i}$ is the smaller one between $\gamma_{S R}^{i}$ and $\gamma_{R D}^{i}$, we can write its $\mathrm{CDF}$ as

$$
F_{\gamma_{e q}^{i}}\left(\gamma_{e q}^{i}\right)=1-e^{-\gamma_{e q}^{i} / \bar{\gamma}_{S R}} e^{-\gamma_{e q}^{i} / \bar{\gamma}_{R D}}=1-e^{-\gamma_{e q}^{i} / \bar{\gamma}_{e q}},
$$

where $\bar{\gamma}_{e q}=\left(\lambda(1-\lambda) \sigma_{2}^{2} \sigma_{3}^{2} /\left(\lambda \sigma_{2}^{2}+(1-\lambda) \sigma_{3}^{2}\right)\right) \bar{\gamma}$. Setting derivative of (19) with respect to $\gamma_{e q}^{i}$, the PDF of $\gamma_{e q}^{i}$ can be derived as

$$
p_{\gamma_{e q}^{i}}\left(\gamma_{e q}^{i}\right)=\frac{1}{\bar{\gamma}_{e q}} e^{-\gamma_{e q}^{i} / \bar{\gamma}_{e q}} .
$$

Note that (10) suggests that $\gamma_{e q}^{J}$ is essentially the largest order statistic of $\gamma_{e q}^{i}$; hence, by utilizing [41, Equation (2.1.1)], we can obtain the CDF in (18); then differentiating it with respect to $\gamma_{e q}^{J}$ and with the help of the binomial theorem, the PDF (17) can be derived. 
3.2. Outage Probability. The outage probability is defined as the probability that the end-to-end SNR drops below a certain threshold $\gamma_{t h}$. With the help of (12), the outage probability can be rewritten as

$$
P_{\text {out }}=\operatorname{Pr}\left\{\gamma_{S D}+\gamma_{e q}^{J}<\gamma_{t h}\right\}
$$

A closed-form outage probability of the network can be derived as the following theorem.

Theorem 2. The outage probability of the RSDDCT-DetF network is given by

$$
P_{\text {out }}=\sum_{k=0}^{N_{R}} C_{N_{R}}^{k} \frac{(-1)^{k} \bar{\gamma}_{e q}}{k \bar{\gamma}_{S D}-\bar{\gamma}_{e q}}\left(e^{-\gamma_{t h} / \bar{\gamma}_{S D}}-e^{-k \gamma_{t h} / \bar{\gamma}_{e q}}\right),
$$

where $\bar{\gamma}_{S D}$ and $\bar{\gamma}_{e q}$ are the average SNR of the direct and equivalent links, respectively.

Proof. By utilizing the law of total probability, the outage probability $(21)$ can be calculated as $P_{\text {out }}=\int_{0}^{\gamma_{\text {th }}} p_{\gamma_{\text {SD }}}(\gamma)$ $F_{\gamma_{e q}^{J}}\left(\gamma_{t h}-\gamma\right) d \gamma$; substitute (15) and (17) into (21); and, with the help of the binomial theorem, Theorem 2 can be derived.

3.3. Average Bit Error Rate. We now turn our attention to the average BER performance of the network. According to [42, Equation (12.1-13)], the conditional BER of the differential
BPSK modulation with $L$-channel diversity reception is given by

$$
P_{b}\left(\gamma_{T}\right)=\frac{1}{2^{2 L-1}} e^{-\gamma_{T}} \sum_{n=0}^{L-1} c_{n} \gamma_{T}^{n}
$$

where $\gamma_{T}$ is the instantaneous total SNR at the receiver and $c_{n}$ is defined as

$$
c_{n}=\frac{1}{n !} \sum_{k=0}^{L-1-n}\left(\begin{array}{c}
2 L-1 \\
k
\end{array}\right) \text {. }
$$

With the help of (5) and (12), the conditional BER of the RSDDCT-DetF network with DDBPSK can be approximated by the conditional BER of the 2-channel reception DBPSK, which arrives at

$$
P_{b}=\frac{1}{8}\left(4+\gamma_{T}^{\prime}\right) e^{-\gamma_{T}^{\prime}},
$$

where $\gamma_{T}^{\prime}=\left(\gamma_{S D}+\gamma_{e q}^{J}\right) / 2-1 / 2$ denotes the total instantaneous $\mathrm{SNR}$ of the equivalent single-differential signals.

Theorem 3. The closed-form average BER of the RSDDCTDetF network with DDBPSK modulation is given by

$$
\begin{aligned}
\bar{P}_{b}=\exp \left(\frac{1}{2}\right)\left[\frac{14+9 \bar{\gamma}_{S D}}{8\left(\bar{\gamma}_{S D}+2\right)^{2}} \frac{N_{R} !}{\prod_{k=1}^{N_{R}}\left(\bar{\gamma}_{e q} / 2+k\right)}+\frac{N_{R} \bar{\gamma}_{e q}}{8\left(\bar{\gamma}_{S D}+2\right)}\right. \\
\left.\quad \times \sum_{k=0}^{N_{R}-1} C_{N_{R}-1}^{k} \frac{(-1)^{k}}{\left(k+1+\bar{\gamma}_{e q} / 2\right)^{2}}\right] .
\end{aligned}
$$

Proof. Due to the independence of $\gamma_{S D}$ and $\gamma_{e q}^{J}$, the average BER can be obtained by averaging (25) with respect to $\gamma_{S D}$ and $\gamma_{e q}^{J}$, respectively, as follows:

$$
\begin{aligned}
& \bar{P}_{b}=\underbrace{\frac{1}{16} \exp \left(\frac{1}{2}\right) \int_{0}^{\infty} \overbrace{\int_{0}^{\infty}\left(7+\gamma_{S D}\right) e^{-\gamma_{S D} / 2} d \gamma_{S D}}^{I_{1}} e^{-\gamma_{e q}^{J} / 2} p_{\gamma_{S D}}\left(\gamma_{S D}\right) p_{\gamma_{e q}^{J}}\left(\gamma_{e q}^{J}\right) d \gamma_{e q}^{J}}_{\bar{P}_{b}^{1}} \\
& +\underbrace{\frac{1}{16} \exp \left(\frac{1}{2}\right) \int_{0}^{\infty} \overbrace{\int_{0}^{\infty} e^{-\gamma_{S D} / 2} p_{\gamma_{S D}}^{I_{2}}\left(\gamma_{S D}\right) d \gamma_{S D}} \gamma_{e q}^{J} e^{-\gamma_{e q}^{J} / 2} p_{\gamma_{e q}^{J}}\left(\gamma_{e q}^{J}\right) d \gamma_{e q}^{J}}_{\bar{P}_{b}^{2}} .
\end{aligned}
$$

We first calculate the first part as

$$
\begin{aligned}
\bar{P}_{b}^{1}= & \frac{1}{16} \exp \left(\frac{1}{2}\right) \overbrace{\frac{28+18 \bar{\gamma}_{S D}}{I_{1}}}^{\left.\bar{\gamma}_{S D}+2\right)^{2}} \frac{N_{R}}{\bar{\gamma}_{e q}} \\
& \times \int_{0}^{\infty}\left(1-e^{-\gamma_{e q}^{J} / \bar{\gamma}_{e q}}\right)^{N_{R}-1} e^{-\left(\left(\bar{\gamma}_{e q}+2\right) / 2 \bar{\gamma}_{e q}\right) \gamma_{e q}^{J}} d \gamma_{e q}^{J}
\end{aligned}
$$

$$
\begin{aligned}
& =\exp \left(\frac{1}{2}\right) \frac{14+9 \bar{\gamma}_{S D}}{8\left(\bar{\gamma}_{S D}+2\right)^{2}} \frac{N_{R} \Gamma\left(N_{R}\right) \Gamma\left(1+\bar{\gamma}_{e q} / 2\right)}{\Gamma\left(N_{R}+1+\bar{\gamma}_{e q} / 2\right)} \\
& =\exp \left(\frac{1}{2}\right) \frac{14+9 \bar{\gamma}_{S D}}{8\left(\bar{\gamma}_{S D}+2\right)^{2}} \frac{N_{R} !}{\prod_{k=1}^{N_{R}}\left(\bar{\gamma}_{e q} / 2+k\right)},
\end{aligned}
$$


where the second equality is derived by using [43, Equation (3.312)] and $\Gamma(x)=\int_{0}^{\infty} e^{-t} t^{x-1} d t$ is the Gamma function. On the other hand, substituting (15) and (17), with the help of [43, Equation (3.351)], the second part of $\bar{P}_{b}$ can be simplified into

$$
\begin{aligned}
& \bar{P}_{b}^{2}=\frac{1}{16} \exp \left(\frac{1}{2}\right) \overbrace{\frac{2}{\bar{\gamma}_{S D}+2}}^{I_{2}} \frac{N_{R}}{\bar{\gamma}_{e q}}
\end{aligned}
$$

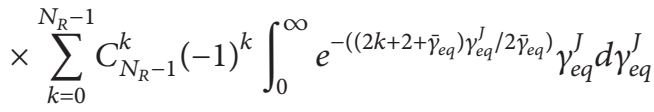

$$
\begin{aligned}
& =\exp \left(\frac{1}{2}\right) \frac{N_{R} \bar{\gamma}_{e q}}{8\left(\bar{\gamma}_{S D}+2\right)} \\
& \times \sum_{k=0}^{N_{R}-1}(-1)^{k} C_{N_{R}-1}^{k} \frac{1}{\left(k+1+\bar{\gamma}_{e q} / 2\right)^{2}} .
\end{aligned}
$$
(29).

To this end, Theorem 3 is obtained by combining (28) and

Note that Theorems 2 and 3 present accurate expressions for the outage probability and average BER of a network with arbitrary number of relay sensors, and the formulas (22) and (26) only involve standard functions which can be directly calculated, thereby providing fast and efficient means to evaluate the system performance.

\section{Asymptotic Behaviors Analysis}

Although the expressions for the outage probability and average BER derived in Theorems 2 and 3 enable numerical evaluation of the system performance and may not be computationally intensive, they do not offer physical insights into the impact of the system parameters, for example, fading parameters and number of relay sensors. To circumvent this, we now focus on the high SNR regime to analyze the asymptotic behaviors of the outage probability and average BER.

\subsection{Asymptotic Outage Probability}

Corollary 4. The asymptotic outage probability of the RSDDCT-DetF network in the high SNR regime is given by

$$
P_{\text {out }}^{\infty}=C_{1}\left(\gamma_{t h}, N_{R}, \sigma_{1}, \sigma_{e q}\right) \frac{1}{\bar{\gamma}^{N_{R}+1}},
$$

where $C_{1}\left(\gamma_{t h}, N_{R}, \sigma_{1}, \sigma_{e q}\right)=\gamma_{t h}^{N_{R}+1} /\left(N_{R}+1\right) \lambda \sigma_{1}^{2} \sigma_{e q}^{2 N_{R}}$, with $\sigma_{e q}^{2}=\lambda(1-\lambda) \sigma_{2}^{2} \sigma_{3}^{2} /\left(\lambda \sigma_{2}^{2}+(1-\lambda) \sigma_{3}^{2}\right)$.
Proof. Making use of the Maclaurin series expansion of the exponential function, $p_{\gamma_{S D}}\left(\gamma_{S D}\right)$ and $F_{\gamma_{e q}^{J}}\left(\gamma_{e q}^{J}\right)$ can be approximated in the high SNR region as

$$
\begin{gathered}
p_{\gamma_{S D}}\left(\gamma_{S D}\right) \approx \frac{1}{\bar{\gamma}_{S D}}-\frac{\gamma_{S D}}{\bar{\gamma}_{S D}^{2}}, \\
F_{\gamma_{e q}^{J}}\left(\gamma_{e q}^{J}\right) \approx\left(\frac{\gamma_{e q}^{J}}{\bar{\gamma}_{e q}}\right)^{N_{R}},
\end{gathered}
$$

where the high-order terms are ignored. Substituting (31) into (22), the outage probability can be rewritten as

$$
\begin{aligned}
P_{\text {out }}^{\infty} & =\frac{1}{\bar{\gamma}_{S D} \bar{\gamma}_{e q}^{N_{R}}} \sum_{k=0}^{N_{R}} C_{N_{R}}^{k}(-1)^{k}\left(\frac{\gamma_{t h}^{N_{R}+1}}{k+1}-\frac{\gamma_{t h}^{N_{R}+2}}{(k+2) \bar{\gamma}_{S D}}\right) \\
& =\frac{\gamma_{t h}^{N_{R}+1}}{\left(N_{R}+1\right) \bar{\gamma}_{S D} \bar{\gamma}_{e q}^{N_{R}}}-\frac{\gamma_{t h}^{N_{R}+2}}{\left(N_{R}+2\right)\left(N_{R}+1\right) \bar{\gamma}_{S D}^{2} \bar{\gamma}_{e q}^{N_{R}}},
\end{aligned}
$$

where we have utilized the identity $\sum_{k=0}^{N_{R}} C_{N_{R}}^{k}(-1)^{k} /(k+$ $m)=N_{R} ! / \prod_{k=0}^{N_{R}}(k+m)$ [28] to derive the second equality. Substituting $\bar{\gamma}_{S D}=\lambda \sigma_{1}^{2} \bar{\gamma}$ and $\bar{\gamma}_{e q}=\left(\lambda(1-\lambda) \sigma_{2}^{2} \sigma_{3}^{2} /\left(\lambda \sigma_{2}^{2}+(1-\right.\right.$ ג) $\left.\left.\sigma_{3}^{2}\right)\right) \bar{\gamma}$ in (32), we can rewrite the outage probability as

$$
\begin{aligned}
& P_{\text {out }}^{\infty}=\frac{\gamma_{t h}^{N_{R}+1}}{\underbrace{\left(N_{R}+1\right) \lambda \sigma_{1}^{2} \sigma_{e}^{2 N_{R}}}_{C_{1}\left(\gamma_{t h}, N_{R}, \sigma_{1}, \sigma_{e q}\right)}} \frac{1}{\bar{\gamma}^{N_{R}+1}} \\
& -\frac{\gamma_{t h}^{N_{R}+2}}{\left(N_{R}+1\right)\left(N_{R}+2\right) \lambda^{2} \sigma_{1}^{4} \sigma_{e q}^{2 N_{R}}} \frac{1}{\bar{\gamma}^{N_{R}+2}},
\end{aligned}
$$

where $\sigma_{e q}^{2}=\lambda(1-\lambda) \sigma_{2}^{2} \sigma_{3}^{2} /\left(\lambda \sigma_{2}^{2}+(1-\lambda) \sigma_{3}^{2}\right)$. Note that the second term in (33) can be ignored when $\bar{\gamma}$ is large enough, which yields Corollary 4 .

\subsection{Asymptotic Average Bit Error Rate}

Corollary 5. The asymptotic average BER of the RSDDCTDetF network with DDBPSK modulation at high SNRs is given by

$$
\bar{P}_{b}^{\infty}=C_{2}\left(N_{R}, \sigma_{1}, \sigma_{2}, \sigma_{3}\right) \frac{\left[\lambda \sigma_{2}^{2}+(1-\lambda) \sigma_{3}^{2}\right]^{N_{R}}}{\lambda^{N_{R}+1}(1-\lambda)^{N_{R}}} \frac{1}{\bar{\gamma}^{N_{R}+1}},
$$

where $C_{2}\left(N_{R}, \sigma_{1}, \sigma_{2}, \sigma_{3}\right)=\exp (1 / 2) 2^{N_{R}-3} N_{R} !((9+$ $\left.\left.2 N_{R}\right) / \sigma_{1}^{2} \sigma_{2}^{2 N_{R}} \sigma_{3}^{2 N_{R}}\right), N_{R}$ is the number of relay sensors, and $\bar{\gamma}$ denotes the average SNR.

Proof. It is obvious that the first part of $\bar{P}_{b}$ can be approximated at sufficiently high SNRs as

$$
\bar{P}_{b}^{1 \infty}=\exp \left(\frac{1}{2}\right) \frac{9}{8 \bar{\gamma}_{S D}} N_{R} ! 2^{N_{R}}\left(\bar{\gamma}_{e q}\right)^{-N_{R}} .
$$


To obtain the asymptotic expression of $\bar{P}_{b}^{2}$, we first denote the sum term of (29) by $I$, as

$$
I=\sum_{k=0}^{N_{R}-1}(-1)^{k} C_{N_{R}-1}^{k} \frac{1}{\left(k+1+\bar{\gamma}_{e q} / 2\right)^{2}} .
$$

Letting $\widehat{\gamma}=1+\bar{\gamma}_{e q} / 2$, after some manipulations, we have

$$
\begin{aligned}
I & =\sum_{k=0}^{N_{R}-1}(-1)^{k} C_{N_{R}-1}^{k} \frac{1}{(k+\widehat{\gamma})^{2}} \\
& =\sum_{m=N_{R}}^{\infty}(-1)^{m-1} m \widehat{\gamma}^{-(m+1)} \sum_{k=0}^{N_{R}-1}(-1)^{k} C_{N_{R}-1}^{k} k^{m-1},
\end{aligned}
$$

where the second equality is derived in a way similar to [27, Equation (31)]. By using [43, Equation (0.154.4)] to calculate the sum term, (37) can be approximated at high SNRs as

$$
\begin{aligned}
I \approx & (-1)^{N_{R}-1} N_{R} \widehat{\gamma}^{-\left(N_{R}+1\right)} \\
& \times \sum_{k=0}^{N_{R}-1}(-1)^{k} C_{N_{R}-1}^{k} k^{N_{R}-1}=N_{R} ! \widehat{\gamma}^{-\left(N_{R}+1\right)} .
\end{aligned}
$$

Recall that $\hat{\gamma} \approx \bar{\gamma}_{e q} / 2$; we then have $I \approx N_{R} ! 2^{N_{R}+1}$ $\left(\bar{\gamma}_{e q}\right)^{-\left(N_{R}+1\right)}$; thus the second part of $\bar{P}_{b}$ can be approximated as

$$
\bar{P}_{b}^{2 \infty}=\frac{N_{R}}{8 \bar{\gamma}_{S D}} N_{R} ! 2^{N_{R}+1}\left(\bar{\gamma}_{e q}\right)^{-N_{R}} .
$$

Adding (35) to (39), we can rewrite the average BER as

$$
\bar{P}_{b}^{\infty}=\exp \left(\frac{1}{2}\right) \frac{1}{\bar{\gamma}_{S D}} 2^{N_{R}-3} N_{R} !\left(9+2 N_{R}\right)\left(\bar{\gamma}_{e q}\right)^{-N_{R}} .
$$

Substituting $\bar{\gamma}_{S D}=\lambda \sigma_{1}^{2} \bar{\gamma}$ and $\bar{\gamma}_{e q}=\left(\lambda(1-\lambda) \sigma_{2}^{2} \sigma_{3}^{2} /\left(\lambda \sigma_{2}^{2}+\right.\right.$ $\left.\left.(1-\lambda) \sigma_{3}^{2}\right)\right) \bar{\gamma}$ in $(40)$, the asymptotic average BER can be expressed as

$$
\begin{aligned}
\bar{P}_{b}^{\infty}= & \frac{\exp \left(\frac{1}{2}\right) 2^{N_{R}-3} N_{R} ! \frac{\left(9+2 N_{R}\right)}{\sigma_{1}^{2} \sigma_{2}^{2 N_{R}} \sigma_{3}^{2 N_{R}}}}{C_{2}\left(N_{R}, \sigma_{1}, \sigma_{2}, \sigma_{3}\right)} \\
& \times \frac{\left[\lambda \sigma_{2}^{2}+(1-\lambda) \sigma_{3}^{2}\right]^{N_{R}}}{\lambda^{N_{R}+1}(1-\lambda)^{N_{R}}} \frac{1}{\bar{\gamma}^{N_{R}+1}} .
\end{aligned}
$$

To this end, Corollary 5 is proved.

Corollaries 4 and 5 demonstrate that a RSDDCT-DetF network with $N_{R}$ relay sensors can achieve a full diversity order of $N_{R}+1$ at sufficiently high SNRs. The above expressions (30) and (34) also reveal straightforwardly the impact of the model parameters on the system performance. More specifically, we can see that, by increasing $N_{R}$ and the sensor nodes' power, the outage probability and average BER will reduce. In addition, we can also observe that the diversity of the MWSN is determined by the number of relay sensors. We will show through numerical evaluation in Section 6 that both the asymptotic outage probability (30) and the average BER (34) tightly correlate with their exact analytical counterparts in the high SNR regime; thus we can precisely predict the system performance through Corollaries 4 and 5 at sufficiently high SNRs.

\section{Optimum Power Allocation}

The optimum power allocation for improving the system performance (e.g., minimizing the outage probability or error probability) has been a very hot research area [24, $26,34]$. As MSNs are generally power-limited systems, it may be of particular importance to investigate the optimum power allocation for MSNs. With this observation in mind, in this section, we address the power allocation issue for the RSDDCT-DetF sensor network under consideration to improve its average BER performance.

Having the closed-form average BER (26), we are about to investigate the power allocation among the source sensor and the relay sensors to minimize the average BER under a total transmit power $P=P_{1}+P_{2}$. The optimization problem of the power allocation can be formulated as

$$
\begin{array}{ll}
\left(P_{1}, P_{2}\right)=\arg \min _{P_{1}, P_{2}} \bar{P}_{b}, \\
\text { s.t. } \quad P_{1}+P_{2}=P, \\
& 0<P_{1}, \quad P_{2}<P .
\end{array}
$$

However, it is generally difficult to directly manipulate the exact average BER expression (26), and the optimum solution can only be derived through exhaustive search. In order to obtain a simple closed-form solution, we choose to look into the high SNR regime and determine the optimum power allocation scheme by use of the asymptotic average BER expression. Note that, given a fading scenario, $C_{2}\left(N_{R}, \sigma_{1}, \sigma_{2}, \sigma_{3}\right) / \bar{\gamma}^{N_{R}+1}$ in (34) is a constant which only depends on the average SNR and the number of relay sensors; therefore, based on the asymptotic average BER expression, the optimization problem of the power allocation can be rewritten as

$$
\begin{aligned}
& \lambda=\arg \min _{\lambda} \frac{\left[\lambda \sigma_{2}^{2}+(1-\lambda) \sigma_{3}^{2}\right]^{N_{R}}}{\lambda^{N_{R}+1}(1-\lambda)^{N_{R}}}, \\
& \text { s.t. } \quad 0<\lambda<1 .
\end{aligned}
$$

Note that (43) is an equation with only one variable, namely, $\lambda$; thus, by differentiating the objective function in (43) with respect to $\lambda$ and setting the derivative equal to zero, we can derive the optimum power allocation factor $\lambda_{\text {op }}$ as follows.

Case $A$. For the scenario $\sigma_{2}^{2}=\sigma_{3}^{2}$, the optimum power allocation factor is

$$
\lambda_{\mathrm{op}}=\frac{N_{R}+1}{2 N_{R}+1} .
$$


Case $B$. For the scenario $\sigma_{2}^{2} \neq \sigma_{3}^{2}$, the optimum power allocation factor is

$$
\lambda_{\mathrm{op}}=\frac{\left[\sigma_{2}^{2}-\left(2 N_{R}+2\right) \sigma_{3}^{2}\right]+\Delta}{2\left(N_{R}+1\right)\left(\sigma_{2}^{2}-\sigma_{3}^{2}\right)},
$$

where $\Delta=\sqrt{\left[\left(2 N_{R}+2\right) \sigma_{3}^{2}-\sigma_{2}^{2}\right]^{2}+4 \sigma_{3}^{2}\left(N_{R}+1\right)^{2}\left(\sigma_{2}^{2}-\sigma_{3}^{2}\right)}$.

We observe from (44) and (45) that the equal power allocation always cannot provide the best average BER performance and the optimum power allocation is dependent on the channel variances, that is, $\sigma_{2}^{2}, \sigma_{3}^{2}$ and the number of relay sensors. More specifically, in the case $\sigma_{2}^{2}=\sigma_{3}^{2}$, the optimum power factor $\lambda_{\text {op }}$ is larger than $1 / 2$ and when $N_{R} \rightarrow$ $+\infty$, the equal power allocation yields the best average BER performance. It can be easily shown that, for the scenario $\sigma_{2}^{2}>\sigma_{3}^{2}$, the best power allocation factor $\lambda_{\text {op }}$ is smaller than $\left(N_{R}+1\right) /\left(2 N_{R}+1\right)$, and when $\sigma_{2}^{2} \gg \sigma_{3}^{2}$, we further have $\lambda_{\text {op }}<$ $1 / 2$, which indicates that more power should be allocated to the relay sensors. On the other hand, when it comes to the scenario $\sigma_{2}^{2}<\sigma_{3}^{2}$, the optimum power factor $\lambda_{\text {op }}$ is larger than $1 / 2$, which suggests that more transmit power should be allocated to the source sensor.

\section{Numerical Results}

In this section, the theoretical results derived in the previous sections are validated by a set of Monte Carlo simulations, where we consider slow Rayleigh fading channels with random CFOs, and the transmission length $L$ is set to 100. Throughout our simulations, we suppose that all the CFOs are mutually independent and uniformly distributed over $\left[-2 \pi f_{d} T, 2 \pi f_{d} T\right]$, where $f_{d} T$ denotes the maximum normalized CFO. Without special explanation, the transmit power is always equally split between the source sensor and the relay sensors, that is, $P_{1}=P_{2}=P / 2$. Note that the SNR refers to the average SNR $\bar{\gamma}$ in the following discussions.

We first examine the outage performance of the RSDDCT-DetF network. Figures 3 and 4 demonstrate the simulated outage probabilities along with the accurate outage probabilities in (22) and their corresponding asymptotic approximations in (30). In our simulations, the predetermined SNR threshold is $\gamma_{t h}=10 \mathrm{~dB}$, and the maximum CFO is $f_{d} T=0.5$. In Figure 3 , we consider three possible numbers of the relay sensors, namely, $N_{R}=1,2,4$, respectively, and the fading gains are equal, that is, $\sigma_{1}^{2}=\sigma_{2}^{2}=\sigma_{3}^{2}=1$. In Figure $4, N_{R}$ is fixed to 3, and four different fading scenarios are investigated. As can be seen from the two figures, the accurate analytical curves match well with the simulation results across the entire SNR range in all the scenarios. Moreover, the proposed asymptotic outage probabilities yield excellent tightness at high SNRs. Likewise, the analytical outage probability expressions can very efficiently predict the exact outage probability. Another observation is that the achieved diversity order is dependent on the number of relay sensors, and it increases when more relay sensors are used. Specifically, about $5 \mathrm{~dB}$ performance improvement can be observed at an outage probability

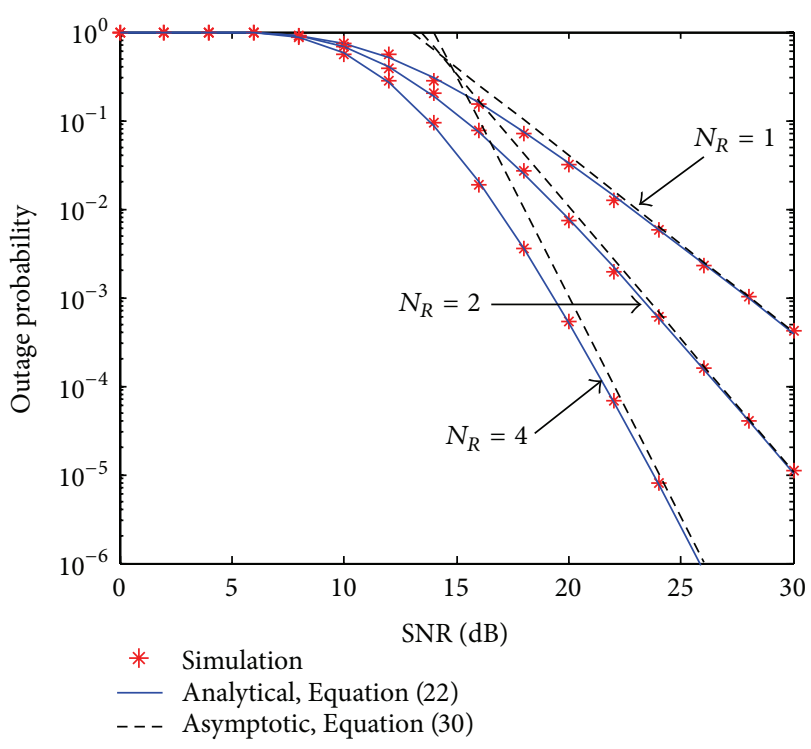

FIGURE 3: Outage probabilities of the RSDDCT-DetF network using different numbers of relay sensors.

of $10^{-3}$ as $N_{R}$ increases from 1 to 2 . In addition, we can obtain a performance gain of about $4 \mathrm{~dB}$ at the same outage probability by increasing $N_{R}$ from 2 to 4 .

In Figures 5 and 6, we proceed to illustrate the average BER performance of the RSDDCT-DetF network. We have performed the simulations in the same scenarios as these of Figures 3 and 4 . In the two figures, the simulated average BER curves are plotted along with the analytical average BER given in (26). Also, the results pertaining to the asymptotic expression for the average BER given by (34) are included in the figures. Note that, for the sake of completeness, the simulated average BER curve of the noncooperative or direct transmission system $\left(N_{R}=0\right)$ is also plotted to serve as a benchmark. It is worth noting that, to fairly compare the performances, we assume that the total transmit powers are equal; in other words, the transmit power of $S$ in the case $N_{R}=0$ is twice that of the other systems. As observed, the analytical expression is in great match with the simulated results, even in the low SNR region in each scenario. In addition, for all the cases, the asymptotic BERs and their other two corresponding curves overlap at high SNRs, which indicate the correctness of our theoretical analysis. In Figure 5, it is clear that each cooperative network outperforms the noncooperative network when SNR exceeds a certain threshold. We can also observe from the two graphs that the achievable diversity order of the network depends on the number of relay sensors, and it is increasing with the number of the potential relay sensors. To be specific, we can observe about $3.5 \mathrm{~dB}$ performance improvement at an average BER of $10^{-3}$ when the number of relay sensors increases from 1 to 2 . And an additional $2.7 \mathrm{~dB}$ gain is obtained at the same average BER when $N_{R}$ increases from 2 to 4 .

Figure 7 compares the average BER performance of the RSDDCT-DetF network and the DM-RS-DetF network with DBPSK modulation, where five different random CFOs are 


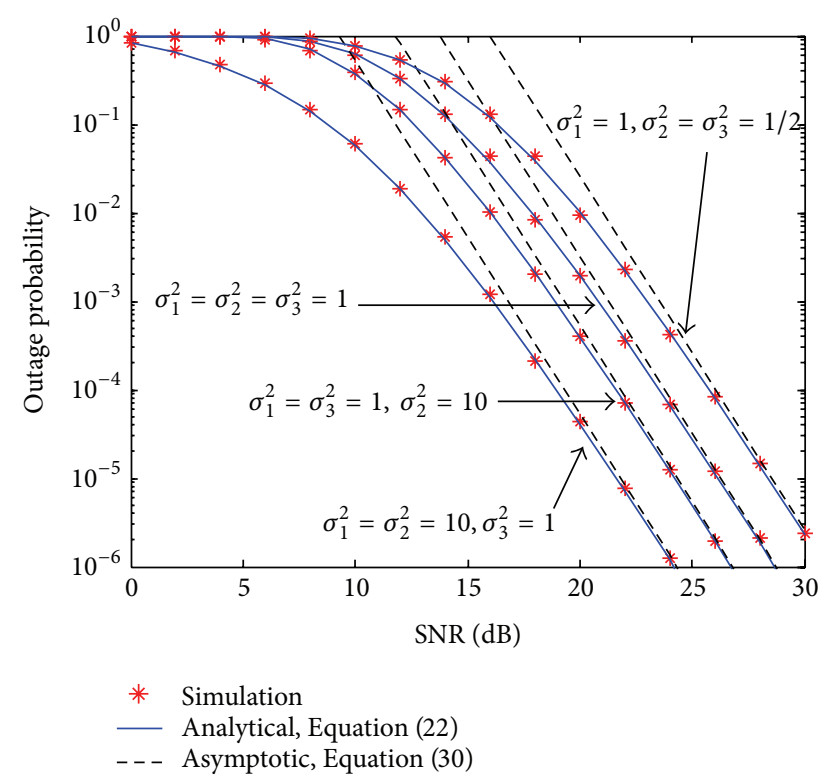

FIGURE 4: Outage probabilities of the RSDDCT-DetF network in different fading scenarios $\left(N_{R}=3\right)$.

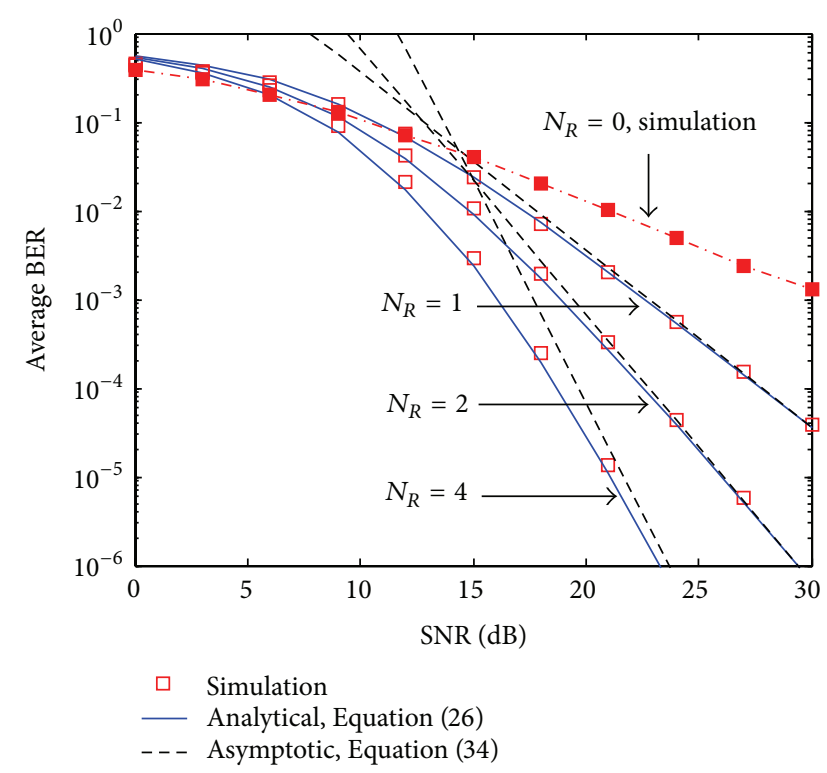

FIGURE 5: Average BER performance of the RSDDCT-DetF network using different numbers of relay sensors.

considered, that is, $f_{d} T=[0,0.125,0.25,0.375,0.5]$. The number of relay sensors is $N_{R}=3$ and the channel variances are $\sigma_{1}^{2}=\sigma_{2}^{2}=\sigma_{3}^{2}=1$. As the RSDDCT-DetF network almost has the same performance under different CFOs, we only plot its one average BER curve. We observe that there is a performance gap of about $3.8 \mathrm{~dB}$ between the RSDDCT-DetF network and the single-differential network in the absence of CFOs $\left(f_{d} T=0\right)$. It is also shown that when random CFOs are involved, the DM-RS-DetF network suffers severe performance degradation while the DD network can achieve excellent performance. For example, when $f_{d} T=0.125$, the performance gap shrinks to $2.2 \mathrm{~dB}$. Furthermore, we can

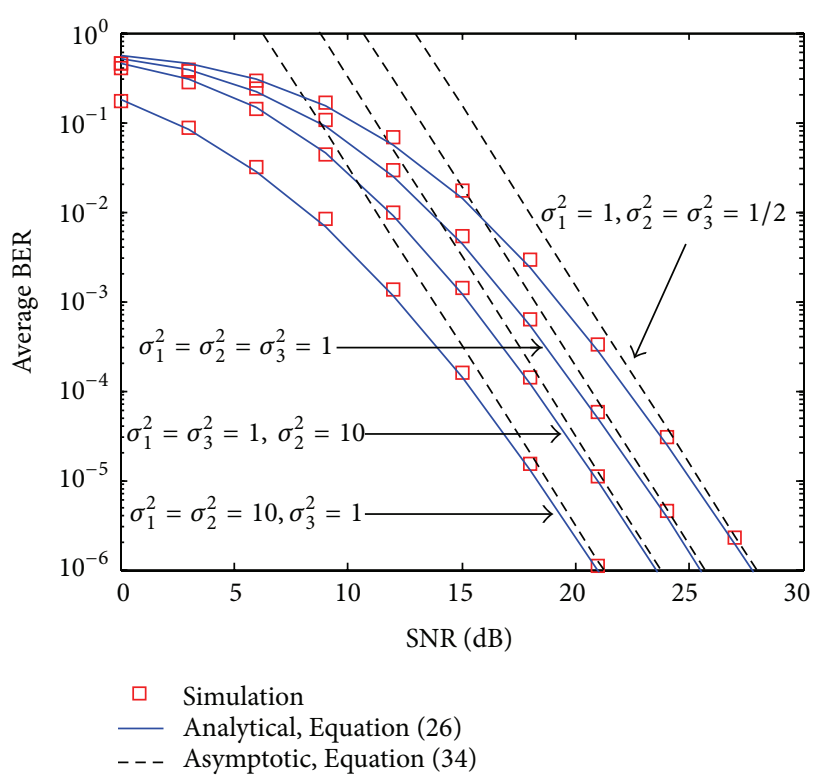

FIGURE 6: Average BER performance of the RSDDCT-DetF network in different fading scenarios $\left(N_{R}=3\right)$.

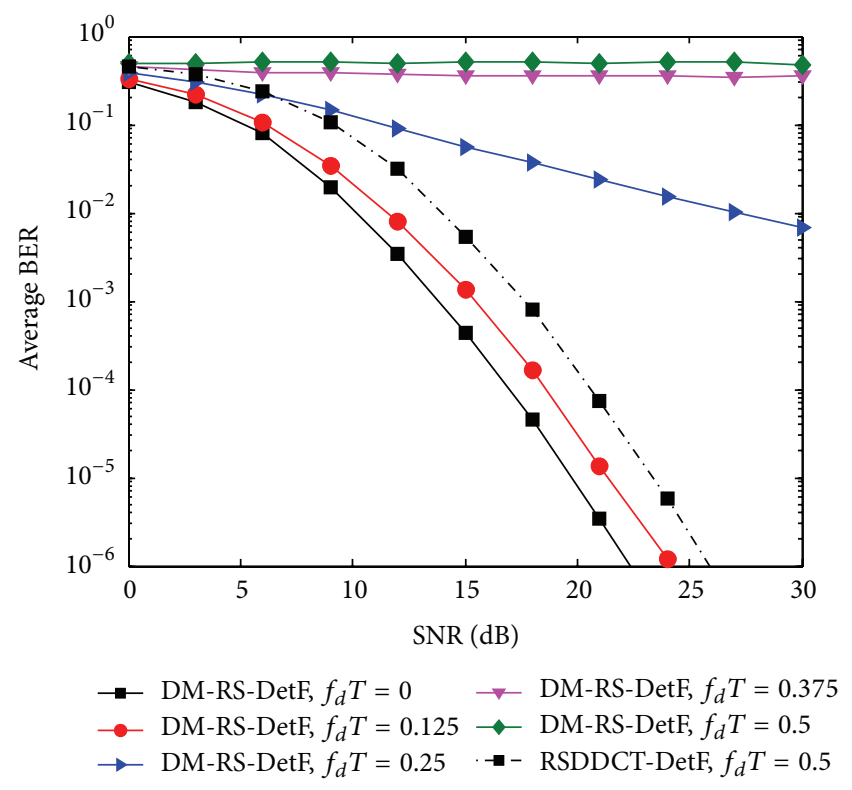

FIGURE 7: Average BER performance comparisons between the RSDDCT-DetF and DM-RS-DetF networks.

observe two collapses from the average BER curves of the DM-RS-DetF network in the cases $f_{d} T=0.375$ and 0.5 .

Figure 8 elaborates on the optimum power allocation factors for the RSDDCT-DetF network with three relay sensors. We consider three different fading scenarios: (A) all the channel gains are equal; that is, $\sigma_{1}^{2}=\sigma_{2}^{2}=\sigma_{3}^{2}=1$ refers to the scenarios where all the relay sensors are located at halfway between the source sensor and the destination sensor; (B) $\sigma_{1}^{2}=\sigma_{3}^{2}=1, \sigma_{2}^{2}=10$ refers to the scenarios where the relay sensors are closer to the source sensor; and (C) $\sigma_{1}^{2}=\sigma_{2}^{2}=1$, $\sigma_{3}^{2}=10$ refers to the scenarios where all the relay sensors 


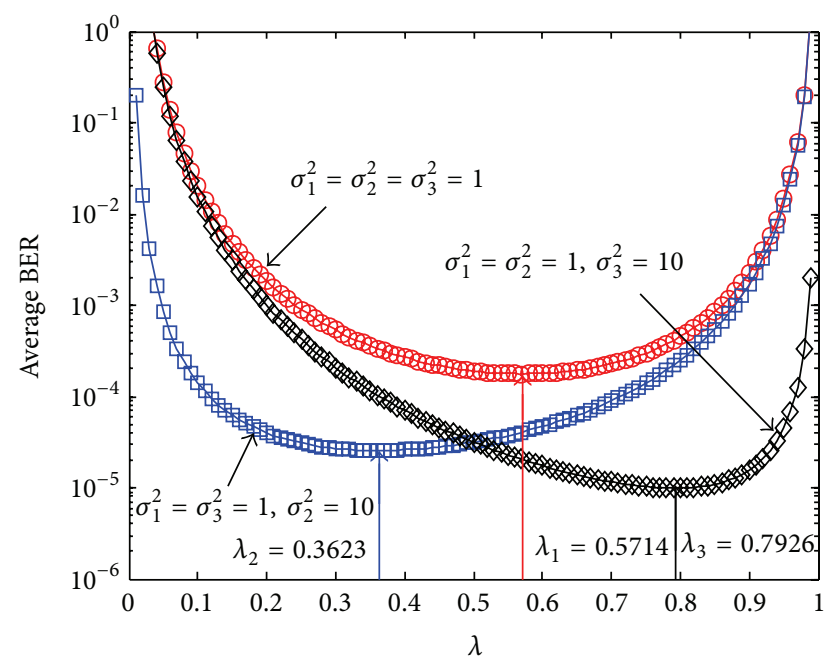

FIGURE 8: Average BER of the RSDDCT-DetF network versus the power allocation factor $\left(N_{R}=3\right)$.

are much closer to the destination sensor. Note that, in the graph, we set $S N R=20 \mathrm{~dB}$, and the analytical average BER curves calculated from (34) are plotted against the power allocation factor $\lambda$, which increases from 0.01 to 0.99 by a step 0.01 . In addition, the optimum power allocation factors obtained from (44) and (45) are also presented. We observe that the numerically derived optimum power factors yield the minimum average BER in all cases. Specifically, in Scenario A, the best power allocation factor is $\lambda_{1}=0.5714$, and, for Scenario B, the best choice of powers is $P_{1}=0.3623 P$ and $P_{2}=0.6377 P$, while, in Scenario C, the best power allocation factor changes to $\lambda_{3}=0.7926$. This is consistent with our analysis in Section 5 .

Finally, we study the benefits of power allocation. Figure 9 shows the average BER performance of the RSDDCT-DetF network with optimum power allocation (OPA) in contrast to that with equal power allocation (EPA). The three scenarios outlined in Figure 8 are investigated. Note that each of the graphs depicts two optimized average BER curves: (1) TOPA, which refers to the average BER of the network with theoretical optimum power allocation (TOPA) given by (44) and (45) and (2) SOPA, which represents the average BER of the network with simulated optimum power allocation (SOPA) derived through exhaustive search. It is clear that in all the three scenarios our derived TOPA and the exhaustively searched SOPA almost yield the same performance. From Figure 9(a), we observe that the OPA outperforms the EPA by about $0.7 \mathrm{~dB}$ at an average BER of $10^{-3}$ in Scenario A. However, when it comes to Scenario B, where the relay sensors are closer to the source sensor node, the OPA has a marginal performance gain against the EPA. The reason is that in this scenario the remodulated signals at the relay sensors are as good as those at the source sensor node; therefore, the EPA scheme almost yields the same performance as the OPA, while, for Scenario C, it is observed in Figure 9(b) that the OPA is about $1.5 \mathrm{~dB}$ superior to the EPA at the same average BER. It is because that using EPA in this scenario leads to low quality of the received signals at the relay sensors, thereby

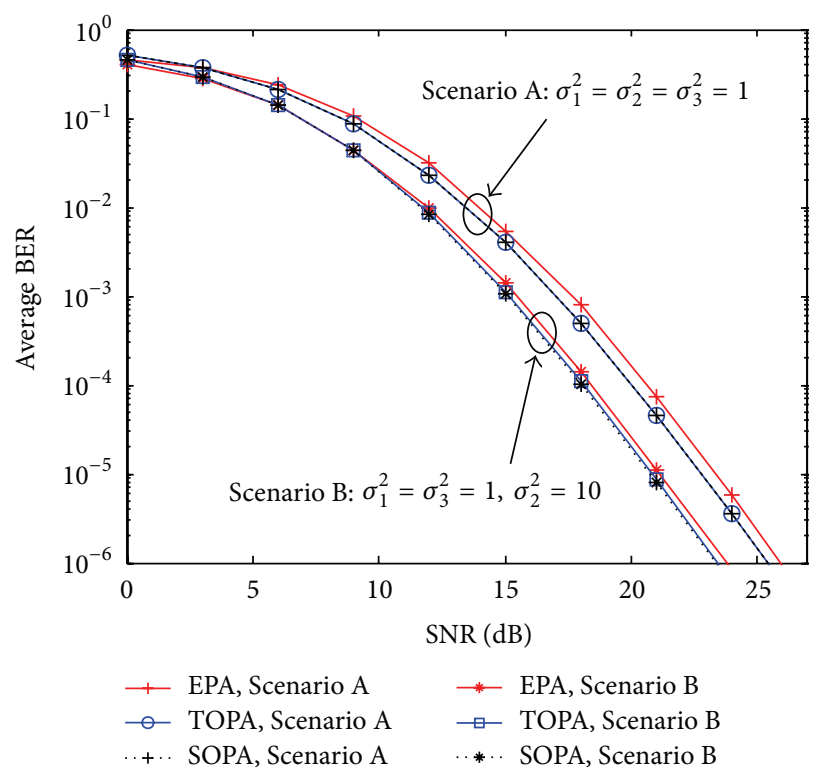

(a)

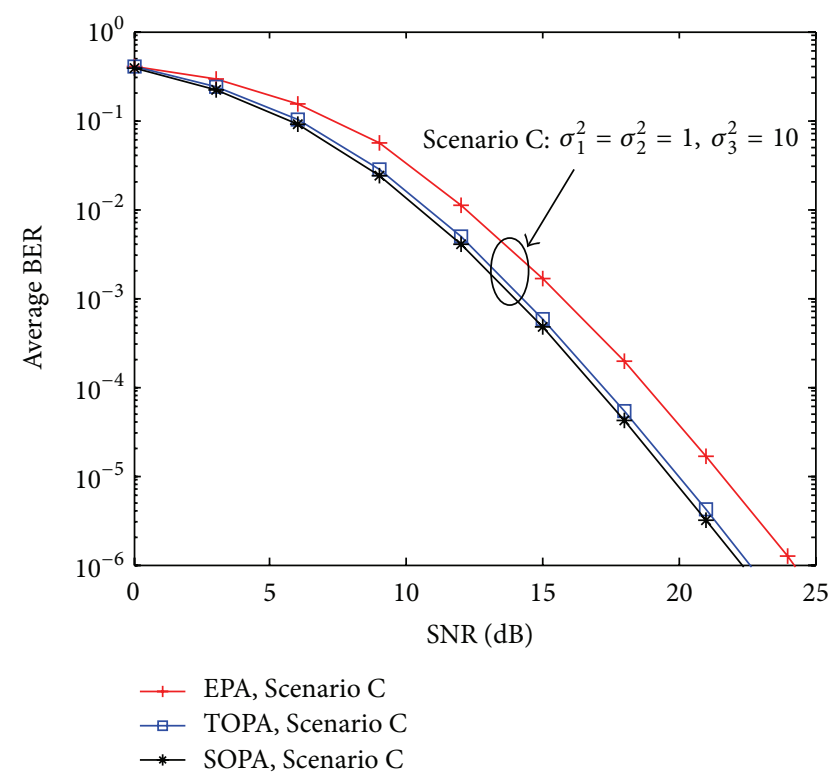

(b)

FIGURE 9: Average BER performance comparisons between the EPA and OPA $\left(N_{R}=3\right)$. (a) Scenarios A and B. (b) Scenario C.

causing high detection errors at the destination sensor. While, with the OPA, more power is allocated to the source sensor, it consequently reduces the detection errors at the relay sensors, which leads to an improvement to the quality of the combined signals at the destination sensor, hence yielding a better average BER performance.

\section{Conclusion}

In this paper, we have proposed and analyzed a robust relay selection based double-differential cooperative transmission (RSDDCT) scheme for MWSNs over Rayleigh fading channels, where all the links are perturbed by random MCFOs. 
In particular, we have derived closed-form expressions for the outage probability and average BER performance of a RSDDCT-DetF network at arbitrary SNRs. Moreover, simplified asymptotic outage probability and average BER expressions in the high SNR regime are deduced, which indicate that a RSDDCT-DetF network consisting of a source sensor, $N_{R}$ relay sensors, and a destination sensor can achieve a full diversity order of $N_{R}+1$ at sufficiently high SNRs. We have shown that in the RSDDCT-DetF networks, the destination sensors are able to detect their data without any knowledge of the channel fading coefficients or MCFOs. It is revealed that the RSDDCT-DetF network is inferior to its single-differential counterpart in the absence of MCFOs; however, the RSDDCT-DetF network performs well over fading channels with random MCFOs, where the singledifferential networks fail to work. We have also investigated the power allocation optimization problem to improve the average BER performance based on the derived analytical expressions. Monte Carlo simulations show that our optimum power allocation strategy provides considerable average BER performance enhancement as compared to the equal power allocation strategy.

\section{Conflict of Interests}

The authors declare that there is no conflict of interests regarding the publication of this paper.

\section{References}

[1] I. F. Akyildiz, W. Su, Y. Sankarasubramaniam, and E. Cayirci, "A survey on sensor networks," IEEE Communications Magazine, vol. 40, no. 8, pp. 102-114, 2002.

[2] J. Yick, B. Mukherjee, and D. Ghosal, "Wireless sensor network survey," Computer Networks, vol. 52, no. 12, pp. 2292-2330, 2008.

[3] J. Rezazadeh, M. Moradi, and A. S. Ismail, "Mobile wireless sensor networks overview," International Journal of Computer Communications and Networks, vol. 2, no. 1, pp. 17-22, 2012.

[4] S. A. Munir, B. Ren, W. Jiao, B. Wang, D. Xie, and J. Ma, "Mobile wireless sensor network: architecture and enabling technologies for ubiquitous computing," in Proceedings of International Conference on Advanced Information Networking and Applications Workshops, pp. 113-120, Niagara Falls, Canada, May 2007.

[5] X. Huang, H. Zhai, and Y. Fang, "Robust cooperative routing protocol in mobile wireless sensor networks," IEEE Transactions on Wireless Communications, vol. 7, no. 12, pp. 5278-5285, 2008.

[6] Y.-C. Wang and Y.-C. Tseng, "Distributed deployment schemes for mobile wireless sensor networks to ensure multilevel coverage," IEEE Transactions on Parallel and Distributed Systems, vol. 19, no. 9, pp. 1280-1294, 2008.

[7] S. A. B. Awwad, C. K. Ng, N. K. Noordin, and M. F. A. Rasid, "Cluster based routing protocol for mobile nodes in wireless sensor network," in Proceedings of of the International Symposium on Collaborative Technologies and Systems (CTS '09), pp. 233-241, Baltimore, Md, USA, May 2009.

[8] N. Meghanathan, "Impact of the Gauss-Markov mobility model on network connectivity, lifetime and hop count of routes for mobile Ad hoc networks," Journal of Networks, vol. 5, no. 5, pp. 509-516, 2010.
[9] J. Rezazadeh, M. Moradi, and A. S. Ismail, "Efficient localization via middle-node cooperation in wireless sensor networks," in Proceedings of International Conference on Electrical, Control, and Computer Engineering (INECCE '11), pp. 410-415, Pahang, Malaysia, June 2011.

[10] L. Karim and N. Nasser, "Reliable location-aware routing protocol for mobile wireless sensor network," IET Communications, vol. 6, no. 14, pp. 2149-2158, 2012.

[11] A. Sendonaris, E. Erkip, and B. Aazhang, "User cooperation diversity-part I: system description," IEEE Transactions on Communications, vol. 51, no. 11, pp. 1927-1938, 2003.

[12] A. Sendonaris, E. Erkip, and B. Aazhang, "User cooperation diversity-part II: implementation aspects and performance analysis," IEEE Transactions on Communications, vol. 51, no. 11, pp. 1939-1948, 2003.

[13] S. Cui, A. J. Goldsmith, and A. Bahai, "Energy-efficiency of MIMO and cooperative MIMO techniques in sensor networks," IEEE Journal on Selected Areas in Communications, vol. 22, no. 6, pp. 1089-1098, 2004.

[14] A. D. Coso, U. Spagnolini, and C. Ibars, "Cooperative distributed MIMO channels in wireless sensor networks," IEEE Journal on Selected Areas in Communications, vol. 25, no. 2, pp. 402-414, 2007.

[15] Z. Zhou, S. Zhou, J.-H. Cui, and S. Cui, "Energy-efficient cooperative communication in a clustered wireless sensor network," IEEE Transactions on Vehicular Technology, vol. 57, no. 6, pp. 3618-3628, 2008.

[16] Z. Rafique, B. C. Seet, and A. Al-Anbuky, "Performance analysis of cooperative virtual MIMO systems for wireless sensor networks," Sensors, vol. 13, no. 5, pp. 7033-7052, 2013.

[17] S. Cho, J.-W. Choi, and C. You, "Adaptive multi-node multiple input and multiple output (MIMO) transmission for mobile wireless multimedia sensor networks," Sensors, vol. 13, no. 10, pp. 13382-13401, 2013.

[18] Z. Zhou, S. Zhou, J.-H. Cui, and S. Cui, "Energy-efficient cooperative communication based on power control and selective single-relay in wireless sensor networks," IEEE Transactions on Wireless Communications, vol. 7, no. 8, pp. 3066-3079, 2008.

[19] C.-L. Wang and S.-J. Syue, "An efficient relay selection protocol for cooperative wireless sensor networks," in Proceedings of of the IEEE Wireless Communications and Networking Conference (WCNC '09), pp. 1-5, Budapest, Hungary, April 2009.

[20] X. Liang, I. Balasingham, and V. C. M. Leung, "Cooperative communications with relay selection for QoS provisioning in wireless sensor networks," in Proceedings of IEEE Global Communications Conference (GLOBECOM '09), pp. 1-8, Honolulu, Hawaii, USA, December 2009.

[21] I. Joe and M. Shin, "An energy-efficient mobile cluster-based approach for vehicular wireless sensor networks," in Proceedings of the 6th International Conference on Networked Computing (INC '10), pp. 1-5, Gyeongju, Korea, May 2010.

[22] M. Quaritsch, K. Kruggl, D. Wischounig-Strucl, S. Bhattacharya, M. Shah, and B. Rinner, "Networked UAVs as aerial sensor network for disaster management applications," Elektrotechnik und Informationstechnik, vol. 127, no. 3, pp. 56-63, 2010.

[23] D. Chen and J. N. Laneman, "Modulation and demodulation for cooperative diversity in wireless systems," IEEE Transactions on Wireless Communications, vol. 5, no. 7, pp. 1785-1794, 2006.

[24] Q. Zhao and H. Li, "Differential modulation for cooperative wireless systems," IEEE Transactions on Signal Processing, vol. 55, no. 5, pp. 2273-2283, 2007. 
[25] T. Wang, A. Cano, G. B. Giannakis, and J. N. Laneman, "High-performance cooperative demodulation with decodeand-forward relays," IEEE Transactions on Communications, vol. 55, no. 7, pp. 1427-1438, 2007.

[26] T. Himsoon, W. P. Siriwongpairat, W. Su, and K. J. R. Liu, "Differential modulations for multinode cooperative communications," IEEE Transactions on Signal Processing, vol. 56, no. 7, pp. 2941-2956, 2008.

[27] J. Yuan, Y. Li, and L. Chu, "Differential modulation and relay selection with detect-and-forward cooperative relaying," IEEE Transactions on Vehicular Technology, vol. 59, no. 1, pp. 261-268, 2010.

[28] Y. Gao, J. Ge, and C. Han, "Performance analysis of differential modulation and relay selection with detect-and-forward cooperative relaying," IEEE Communications Letters, vol. 15, no. 3, pp. 323-325, 2011.

[29] H. Mehrpouyan and S. Blostein, "Synchronization in cooperative networks: estimation of multiple carrier frequency offsets," in Proceedings of IEEE International Conference on Communications (ICC '10), pp. 1-6, Cape Town, South Africa, May 2010.

[30] H. Mehrpouyan and S. D. Blostein, "Bounds and algorithms for multiple frequency offset estimation in cooperative networks," IEEE Transactions on Wireless Communications, vol. 10, no. 4, pp. 1300-1311, 2011.

[31] A. A. Nasir, H. Mehrpouyan, S. D. Blostein, S. Durrani, and R. A. Kennedy, "Timing and carrier synchronization with channel estimation in multi-relay cooperative networks," IEEE Transactions on Signal Processing, vol. 60, no. 2, pp. 793-811, 2012.

[32] A. A. Nasir, H. Mehrpouyan, S. D. Blostein, S. Durrani, and R. A. Kennedy, "Estimation of synchronization parameters in AF cooperative networks," in Proceedings of the IEEE International Conference on Communications (ICC '12), pp. 3713-3717, Ottawa, Canada, June 2012.

[33] Y. B. Okunev, Theory of Phase-Difference Modulation, Moscow, Russia, 1979.

[34] M. R. Bhatnagar, A. Hjørungnes, and L. Song, "Cooperative communications over flat fading channels with carrier offsets: a double-differential modulation approach," EURASIP Journal on Advances in Signal Processing, no. 15, pp. 1-11, 20082008.

[35] M. R. Bhatnagar, A. Hjørungnes, L. Song, and R. Bose, "Doubledifferential decode-and-forward cooperative communications over Nakagami-m channels with carrier offsets," in Proceedings of the IEEE Sarnoff Symposium, pp. 1-5, Princeton, NJ, USA, April 2008.

[36] N. Yi, Y. Ma, and R. Tafazolli, "Doubly differential communication assisted with cooperative relay," in Proceedings of the IEEE Vehicular Technology Conference (VTC Spring '08), pp. 644-647, Singapore, May 2008.

[37] M. R. Bhatnagar and O. Tirkkonen, "PL decoding in double differential modulation based decode-and-forward cooperative system," IEEE Communications Letters, vol. 17, no. 5, pp. 860863, 2013.

[38] C. Kim, S. Heo, Y. Bae, and J. Lee, "Relay selection for amplifyand-forward systems with differential and double-differential modulation," in Proceedings of the IEEE 54th International Midwest Symposium on Circuits and Systems (MWSCAS '11), pp. 1-4, Seoul, Korea, August 2011.

[39] P. Stoica, J. Liu, and J. Li, "Maximum-likelihood double differential detection clarified," IEEE Transactions on Information Theory, vol. 50, no. 3, pp. 572-576, 2004.
[40] M. K. Simon and M.-S. Alouini, Digital Communications Over Fading Channels, John Wiley \& Sons, Hoboken, NJ, USA, 2nd edition, 2005.

[41] H. A. David and H. N. Nagaraja, Order Statistics, John Wiley \& Sons, Hoboken, NJ, USA, 3rd edition, 2003.

[42] J. G. Proakis, Digital Communications, McGraw-Hill, New York, NY, USA, 4th edition, 2001.

[43] I. S. Gradshteyn and I. M. Ryzhik, Table of Integrals, Series, and Products, Elsevier/Academic Press, San Diego, Calif, USA, 7th edition, 2007. 


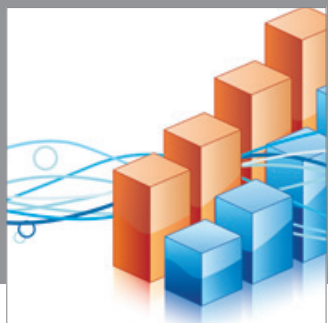

Advances in

Operations Research

mansans

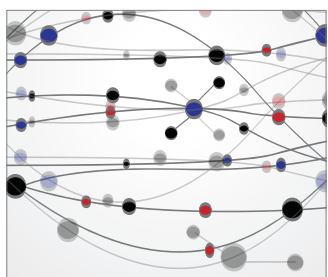

The Scientific World Journal
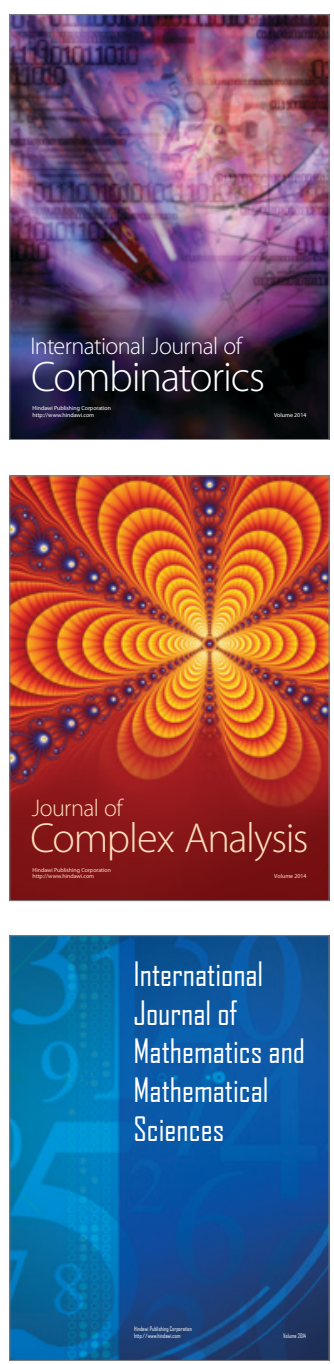
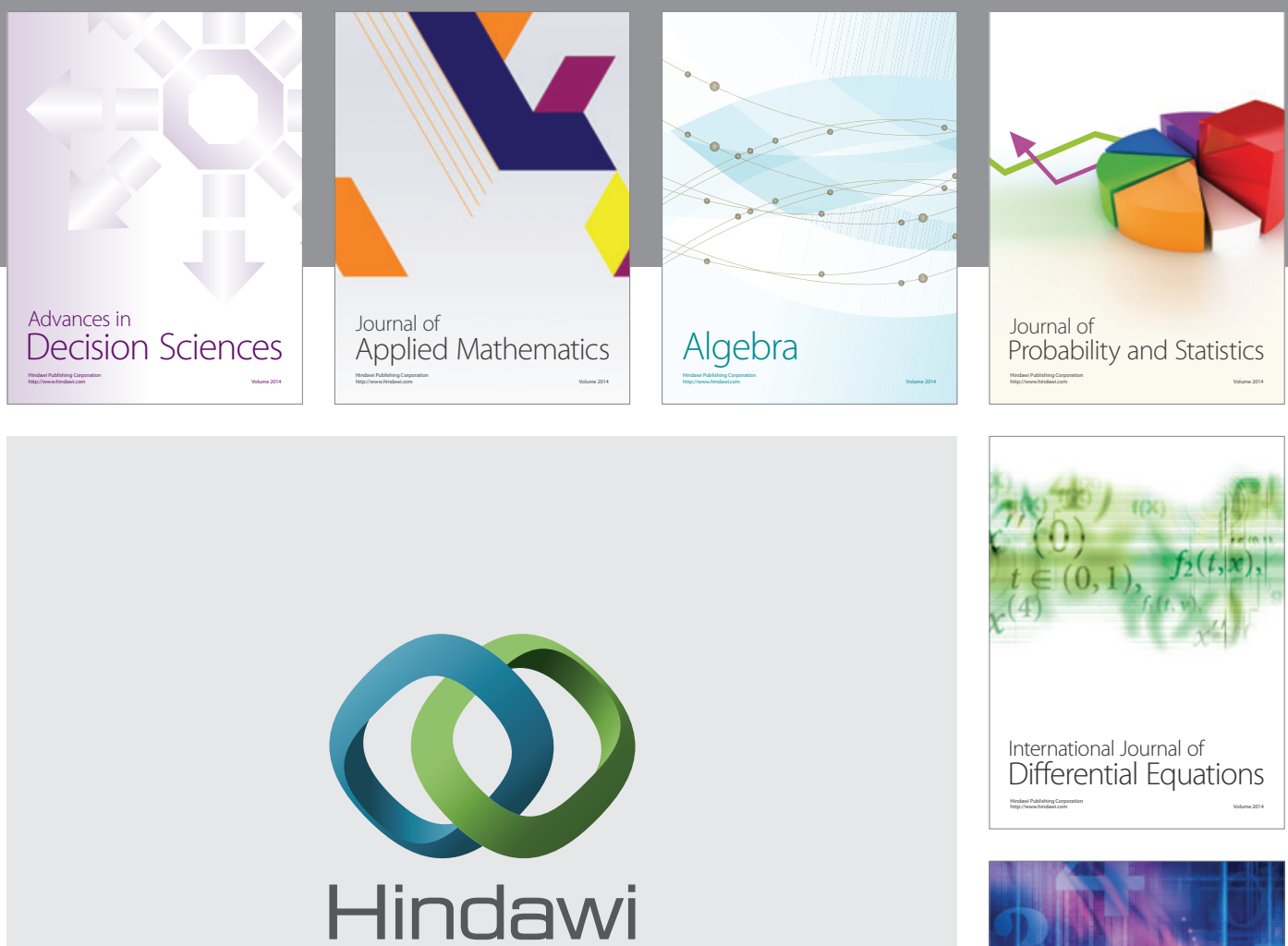

Submit your manuscripts at http://www.hindawi.com
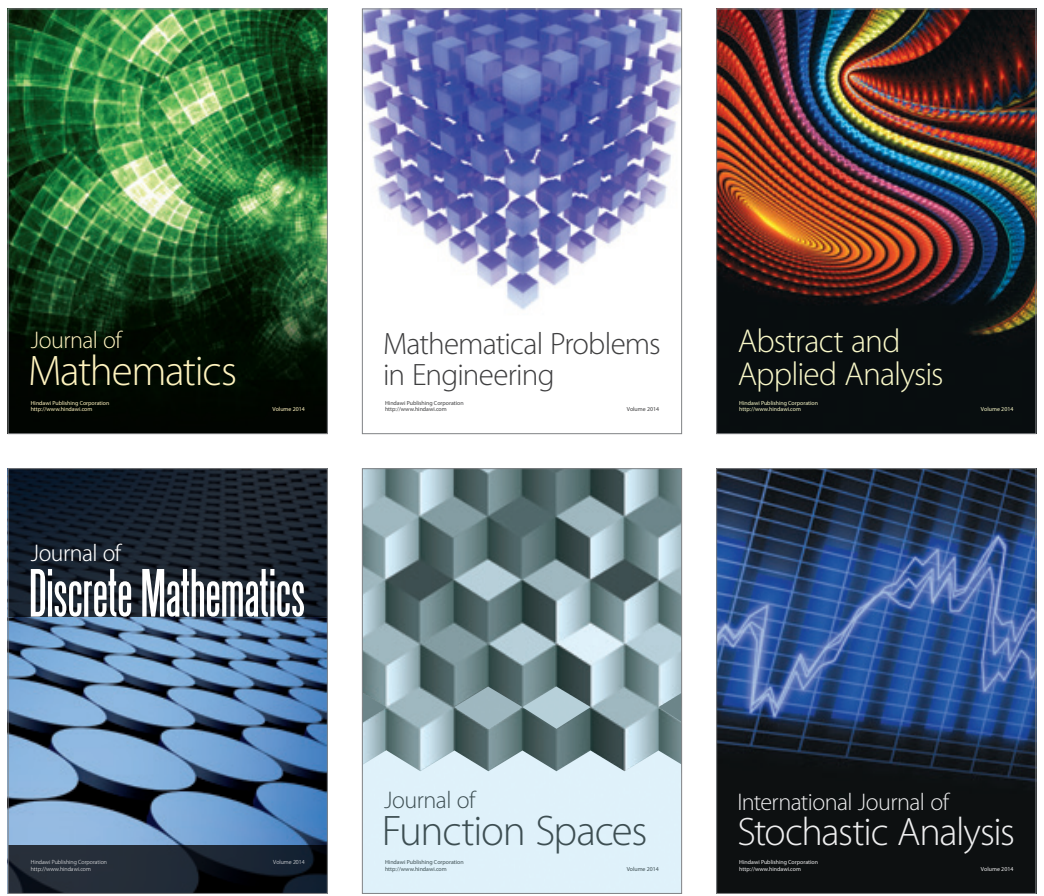

Journal of

Function Spaces

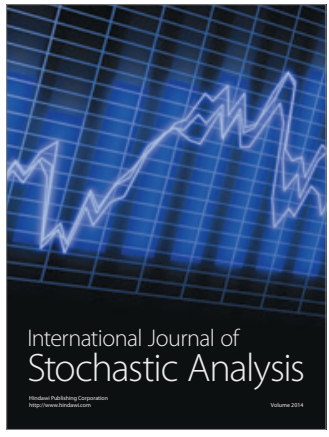

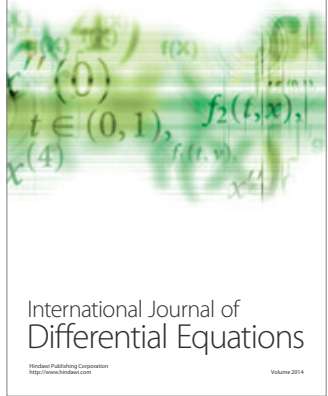
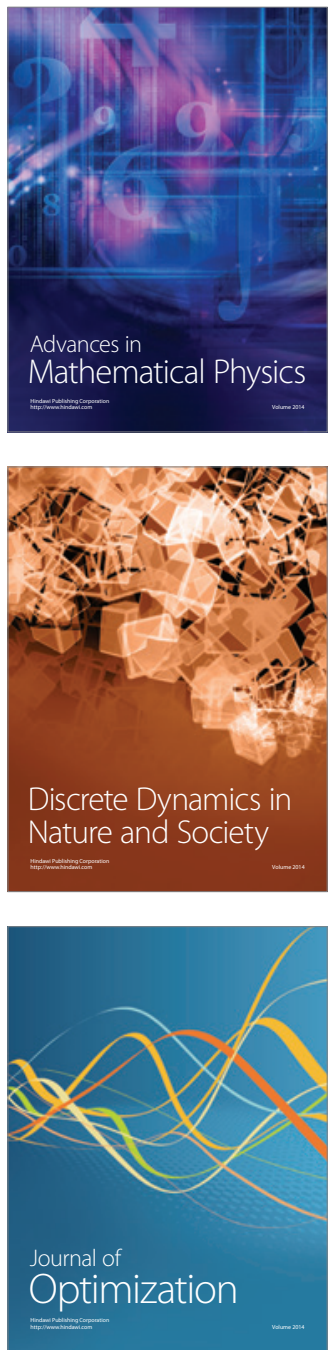